\title{
Características hematológicas y patológicas de cerdos inoculados experimentalmente con el aislado chileno del virus síndrome respiratorio y reproductivo porcino ${ }^{\#}$
}

\author{
Haematological and pathological findings of pigs experimentally inoculated with
}

a Chilean isolate of porcine reproductive and respiratory syndrome virus

\author{
E Ramírez ${ }^{1}$, A Ruiz ${ }^{1}$, A Islas ${ }^{1}$, C Lecocq ${ }^{2}$, L Carrasco ${ }^{3}$, M. Quezada ${ }^{*}$ \\ ${ }^{1}$ Facultad de Medicina Veterinaria, Universidad de Concepción, Av. Vicente Méndez 595, Chillán, Chile. \\ ${ }^{2}$ Subdepartamento Laboratorios y Estación Cuarentenaria Agrícola y Pecuaria, Servicio Agrícola y Ganadero, \\ Av. Bulnes 140, Chile. \\ ${ }^{3}$ Departamento de Anatomía y Anatomía Patológica Comparada, Facultad de Veterinaria, Universidad de Córdoba, \\ Campus de Rabanales, Ctra. Madrid Km. 396, Córdoba, España.
}

\begin{abstract}
SUMMARY
The aims of this study were to characterize the haematological and bone marrow changes, gross and microscopic lesions of pigs experimentally inoculated with the Chilean isolate of porcine reproductive and respiratory syndrome virus. Twelve 3-week-old pigs were divided in 4 groups of 3 , one of which corresponded to the negative control group sacrificed at 0 days post-inoculation (dpi), and the 3 remaining groups corresponded to the inoculated pigs sacrificed at 7, 14 and 21 dpi. For each sampling period blood was collected for complete haemograme and at the necropsy time gross lesions were registered and samples for both bone marrow smears and histopathology were taken. The results of this study revealed haematological alterations characterized by a significant reduction $(\mathrm{P}<0.05)$ in the haematocrit and a significant increase $(\mathrm{P}<0.05)$ in the total leukocyte count associated with an increase in the monocytes and baciliforms. The bone marrow did not show significant variations in the ratio of myeloid to erythroid cells $(P>0.05)$. At the same time, the gross lesions were mild and mainly characterized by the presence of conjunctivitis, periocular edema and a slight increase in the size of the lymph nodes. Microscopic lesions were characterized by the presence of interstitial pneumonia, depletion and necrosis in lymphoid organs, rhinitis, hepatitis, myocarditis and non-purulent encephalitis. These findings suggest that the Chilean isolate of the vPRRS to a strain with a low virulence.
\end{abstract}

Palabras clave: PRRS, histopatología, pulmón.

Key words: PRRS, histopathology, lung.

\section{INTRODUCCION}

El Síndrome Respiratorio y Reproductivo Porcino (PRRS) es una enfermedad de importancia económica en la industria porcina mundial, reportada por primera vez en Estados Unidos en 1987 y en Europa en 1991 (Goyal 1993). El primer aislamiento viral se realizó el año 1991 en Holanda a partir de cultivos de macrófagos alveolares pulmonares (MAPs) (Wensvoort y col 1991). El PRRS es causado por un virus ARN, clasificado dentro del género de los Arterivirus, Familia Arteriviridae, Orden Nidovirales, junto al virus del aumento de la deshidrogenasa láctica del ratón, virus de la arteritis viral equina y virus de la fiebre hemorrágica del simio, los

Aceptado: 29.11.2005

\# Financiado por el Proyecto FONDECYT 1020217.

* Manuel Quezada Orellana, $N^{\circ}$ fax: 042-273201. Correo electrónico:mquezad@udec.cl. Casilla de correo 537, Chillán. cuales presentan varias similitudes incluyendo replicación en macrófagos e infección asintomática persistente (Cavanagh 1997). Los aislados del virus PRRS (vPRRS) se han clasificado en dos serotipos, el americano y el europeo, los cuales presentan diferencias genéticas, antigénicas y biológicas. Estas últimas se traducen en variaciones de virulencia respiratoria y reproductiva (Benfield y col 1999).

El vPRRS causa una enfermedad multisistémica (Rossow y col 1995) y se ha demostrado la presencia del antígeno viral y su capacidad de multiplicación en macrófagos de cornetes nasales, pulmón, nódulos linfáticos, corazón, timo, vasos sanguíneos, bazo, hígado, glándulas adrenales, riñones, intestino, cerebro (Pol y col 1991, Halbur y col 1995, Halbur y col 1996a, Rossow y col 1996a) y testículos (Sur y col 1997); sin embargo, se multiplica preferentemente en MAPs inmaduros o recientemente activados (Molitor y col 1997). Los principales signos clínicos de la enfermedad incluyen cuadros respiratorios en cerdos jóvenes, carac- 
terizados por disnea, hiperapnea y taquipnea (Done y Paton 1995, Rossow y col 1995), así como edema periocular, conjuntivitis y aumento de tamaño de los nódulos linfáticos (Rossow y col 1994, Rossow y col 1995, Done y Paton 1995). A su vez, hay fallas reproductivas en cerdas gestantes caracterizadas principalmente por abortos tardíos (Chistianson y col 1992, Done y Paton 1995, Rossow 1998, Nodelijk 2002).

Los cambios hematológicos más importantes son anemia y un incremento significativo en la relación mieloide: eritroide (M:E) entre los 3 y 21 días postinoculación (dpi) (Halbur y col 2002). Se ha descrito también leucopenia asociada a disminución de linfocitos y monocitos entre los 3 y 7 dpi (Nielsen y Bøtner 1997, Feng y col 2001, Halbur y col 2002).

Las lesiones más comunes se producen en los pulmones y órganos linfoides (Rossow y col 1995), aunque macroscópicamente las lesiones pulmonares resultan poco evidentes (Nodelijk, 2002) ya que pueden ser inaparentes o presentar consolidación difusa en los lóbulos craneales (Rossow y col 1995) asociado, principalmente, a infecciones bacterianas secundarias (Rossow 1998). Los nódulos linfáticos aumentan 2 a 10 veces su tamaño normal (Rossow y col 1995). Histológicamente la lesión más característica de los pulmones corresponde a neumonía intersticial con engrosamiento de los septos, hiperplasia e hipertrofia de los neumocitos tipo II, hiperplasia del tejido linfoide peribronquial y peribronquiolar e infiltrado perivascular linfoplasmocitario (Rossow y col 1994, Done y Paton 1995, Rossow y col 1995, Halbur y col 1996b, Rossow 1998). Las lesiones en nódulos linfáticos y bazo se caracterizan por hiperplasia de centros germinales, depleción y apoptosis (Rossow y col 1995, Halbur y col 1996b, Rossow 1998). También se han descrito rinitis, miocarditis, hepatitis y meningoencefalitis no purulentas (Pol y col 1991, Collins y col 1992, Rossow y col 1995, Halbur y col 1996b, Rossow y col 1996b, Rossow y col 1999).

En Chile, el PRRS fue diagnosticado el año 2000 y actualmente es una enfermedad endémica sometida a un programa de erradicación (Ruiz y col 2003). El presente trabajo se planteó con el fin de caracterizar las manifestaciones hematológicas y lesiones de cerdos inoculados con el aislado chileno del vPRRS.

\section{MATERIAL Y METODOS}

VIRUS. Se utilizó el aislado chileno del vPRRS (cepa 2402), serotipo norteamericano, proporcionado por el Servicio Agrícola y Ganadero (SAG), mantenido en cultivos celulares de línea MARC-145, sometida a dos pasajes y a una concentración de $10^{5,4} \mathrm{TCID}_{50}$ (dosis infectante de cultivo celular). El virus fue inoculado inicialmente en tres cerdos provenientes de núcleos genéticos de alta salud, libres de PRRS, para reactivar su patogenicidad. Los cerdos fueron mantenidos en salas de aislamiento de la Unidad de Control de Productos Biológicos del Departamento de Laboratorios y Estaciones Cuarentenarias Agrícolas y Pecuarias, Complejo Lo Aguirre (Chile) perteneciente al SAG, el cual dispone de las medidas de bioseguridad para evitar su difusión al exterior. De estos animales se obtuvo suero en fase virémica (7 dpi), comprobado por RT-PCR, el cual se filtró utilizando filtros de microporo de $0,2 \mu \mathrm{m}$ y se le adicionaron $100 \mathrm{UI} / \mathrm{ml}$ de penicilina y $100 \mathrm{mg} / \mathrm{ml} \mathrm{de}$ estreptomicina, 24 horas antes de la inoculación de los cerdos del grupo experimental.

CERDOS EXPERIMENTALES E INSTALACIONES. Para este estudio, se utilizaron 12 cerdos híbridos, de ambos sexos ( 5 hembras y 7 machos), de 3 semanas de edad y $6 \mathrm{~kg}$ de peso vivo al inicio de la experiencia. Los animales se obtuvieron del mismo origen que los cerdos utilizados para la reactivación viral y fueron mantenidos en las mismas dependencias anteriormente mencionadas, pertenecientes al SAG. A su llegada, los animales fueron divididos, al azar, en 4 grupos de 3 animales cada uno. Uno de los grupos se utilizó como control y fueron mantenidos en la unidad de aislamiento A. Los tres grupos restantes fueron localizados en la unidad de aislamiento B. Todos los animales permanecieron una semana en aclimatación, manteniéndose en sus respectivas unidades de aislamiento, cada una de las cuales cuenta con corrales adaptados para cerdos, de $6 \mathrm{~m}^{2}$, con piso elevado de concreto parcialmente cubierto con slat, ventilación mecánica y temperatura controlada. Durante este periodo se determinó que todos los animales estaban libres de vPRRS mediante ELISA (Laboratorio IDEXX®) y RT-PCR, así como de patógenos bacterianos más comunes mediante cultivos. Posteriormente, los tres grupos de cerdos localizados en la unidad B, fueron inoculados con $7 \mathrm{ml}$ intranasal y $0,7 \mathrm{ml}$ intramuscular de suero de cerdos virémicos. Los animales del grupo control (unidad A) fueron inoculados por las mismas vías y dosis con PBS estéril. La transmisión de la enfermedad a través del personal fue prevenida a través de medidas de bioseguridad (ducha y cambio de ropa antes del ingreso a las unidades de aislamiento, uso de mascarillas y cofia). Ambas unidades (A y B) fueron equipadas con filtros de aire hepa.

Durante el periodo experimental, todos los cerdos se mantuvieron con alimento sin antibióticos y agua ad libitum. Adicionalmente, se controló en forma diaria la temperatura corporal y se monitorearon los síntomas clínicos, especialmente aquella que indique decaimiento, anorexia, deshidratación, dificultad respiratoria, secreción nasal, eritema de la piel (orejas, abdomen) y síntomas nerviosos.

OBTENCION DE MUESTRAS DE SANGRE. Previo al sacrificio de los animales, se obtuvieron muestras de sangre a $\operatorname{los} 0,7,14$ y 21 dpi a través de una punción en la vena cava anterior, utilizando tubos al vacío con anticoagulante 
(EDTA) y sin anticoagulante. Los tubos con EDTA fueron refrigerados a $4^{\circ} \mathrm{C}$ para realizar hemogramas completos. Con el suero obtenido de las muestras sin anticoagulante se determinaron las proteínas séricas y se realizaron pruebas de ELISA (Laboratorio IDEXX®), siguiendo el protocolo del fabricante, y RT-PCR (Shin y col 1997).

SACRIFICIO, NECROPSIA Y TOMA DE MUESTRAS PARA HISTOPATOLOGIA Y MEDULA OSEA. El grupo control se sacrificó el día $0(n=3)$, dos horas posteriores a la inoculación de todos los animales, con el fin de obtener muestras control para histopatología, inmunohistoquímica y RT-PCR. Por otra parte, los tres grupos de cerdos inoculados fueron sacrificados a $\operatorname{los} 7(n=3), 14(n=3)$ y $21(\mathrm{n}=3)$ dpi, para lo cual se tranquilizaron con acepromazina al $1 \%$ utilizando una dosis de $0,5 \mathrm{mg} / \mathrm{kg}$ de peso vivo vía intramuscular, y se anestesiaron con tiopental sódico diluido al $5 \%$, utilizando una dosis de inducción de $50 \mathrm{mg} / \mathrm{kg}$. Posteriormente, se utilizó el método de perfusión intravascular con tampón fosfato $0,1 \mathrm{M}(\mathrm{pH}$ 7,4 ) a una presión de $80 \mathrm{~mm}$ de mercurio. Por otra parte, se observaron y registraron las lesiones externas presentadas por cada uno de los cerdos sacrificados y se obtuvieron frotis de médula ósea desde las esternebras a través de un corte transversal del esternón. Así también, se obtuvieron muestras de cornetes nasales, tonsila, nódulos linfáticos retrofaríngeos y mediastínico, pulmón (segmento medio del lóbulo cardiaco), bazo, timo, corazón, hígado y encéfalo para histopatología, las cuales fueron fijadas, por 24 a 48 horas, en formol tamponado al $10 \%$.

\section{PROCESAMIENTO DE LAS MUESTRAS}

Hematología. El volumen globular (VG), el recuento total y diferencial de leucocitos fue obtenido a través de los métodos convencionales descritos por Schalm y col (1975). El fibrinógeno se calculó a través de la diferencia entre proteínas plasmáticas totales y proteínas séricas obtenidas a partir del suero de las muestras de sangre sin anticoagulante.

Médula ósea. Los frotis de médula ósea fueron teñidos con solución Giemsa. Para el recuento celular, se utilizó un microscopio de luz con aumento de $1000 \mathrm{X}$ y se contaron 500 células al azar diferenciando aquellas que pertenecen a la línea mieloide y a la línea eritroide. La relación mielode: eritroide (M:E) se obtuvo por la división del total de células mieloides por el total de células eritroides.

Histopatología. Los tejidos fueron procesados utilizando un procesador Shandon (modelo Citadel 1000) con bomba de vacío y un Centro de Inclusión Microm (modelo AP280-2). De los bloques de parafina se realizaron cortes de $4 \mu \mathrm{m}$ con un micrótomo Leica (modelo RM2045), los que fueron teñidos con hematoxilina y eosina. Posteriormente los tejidos fueron observados y fotografiados con un microscopio Zeiss (modelo Axioskop 50).

ANALISIS ESTADISTICO. Los resultados del estudio hematológico y de médula ósea fueron sometidos a análisis de varianza de una vía para determinar diferencias entre las medias de los grupos, con un $95 \%$ de confianza. Cuando el análisis resultó significativo, las medias de los grupos fueron testeadas a través del método de comparación de medias Least-Significant-Differences (LSD) para establecer diferencias significativas. Para este procedimiento se utilizó el programa computacional SPSS versión 10,0 para Windows (SPSS Inc 1989-1999)

\section{RESULTADOS}

En el 100\% de los cerdos inoculados se detectó el virus, mediante RT-PCR, en muestras de suero sanguíneo a los 7 y 14 dpi, disminuyendo estos valores a $67 \%$ a los 21 dpi. Los anticuerpos anti-vPRRS fueron detectados por ELISA desde los 14 dpi en el 50\% de los cerdos y en el $100 \%$ a los 21 dpi. A través de la inmunohistoquímica se detectó inmunorreacción en cornetes nasales, tonsila, nódulos linfáticos retrofaringeos y mediastínicos, parénquima pulmonar, bazo, timo, corazón e hígado afectando preferentemente macrófagos residentes de los tejidos analizados. Los cerdos controles fueron negativos para las tres pruebas.

ESTUDIO HEMATOLOGICO. Se observó una disminución del VG $(\mathrm{P}<0,05)$ en los cerdos de los grupos inoculados. Las PPT y fibrinógeno no presentaron cambios significativos $(\mathrm{P}>0,05)$; sin embargo, se observó una tendencia a aumentar en el tiempo. El número de leucocitos aumentó asociado al incremento en el recuento de monocitos $(\mathrm{P}<0,05)$. Los linfocitos, neutrófilos y eosinófilos no presentaron cambios significativos $(\mathrm{P}>0,05)$ (cuadro 1).

ANALISIS DE MEDULA OSEA. Las medias de la relación M:E de los cerdos inoculados y controles no presentaron modificaciones relevantes entre grupos (cuadro 2).

MANIFESTACIONES CLINICO-PATOLOGICAS. Se observó conjuntivitis en dos cerdos a los 7 dpi, un cerdo a los 14 dpi y en dos cerdos a los 21 dpi. Ningún animal presentó aumento de temperatura corporal ni sintomatología respiratoria. Los cerdos sacrificados a los 21 dpi presentaron retraso en el crecimiento, pelaje hirsuto, edema periocular y leve aumento de tamaño de los nódulos linfáticos inguinales, mandibulares, retrofaríngeos, presternales y mediastínicos. Ningún cerdo presentó lesiones neumónicas evidentes. 
Cuadro 1. Promedios $( \pm \mathrm{DE})$ de los valores hematológicos obtenidos de los cerdos controles a los 0 dpi y en los cerdos inoculados con el aislado chileno del vPRRS, obtenidas a los $0,7,14$ y 21 dpi. 7, 14 and $21 \mathrm{dpi}$.

Means $( \pm \mathrm{SD})$ of haematological values obtained from the control pigs and pigs inoculated whit Chilean PRRSv isolates obtained at 0 ,

\begin{tabular}{ccccccccc}
\hline $\begin{array}{c}\text { Grupos } \\
\text { DPI }\end{array}$ & $\begin{array}{c}\text { VG } \\
(\%)\end{array}$ & $\begin{array}{c}\text { PPT } \\
(\mathrm{g} / \mathrm{L})\end{array}$ & $\begin{array}{c}\text { Fibrinógeno } \\
(\mathrm{g} / \mathrm{L})\end{array}$ & $\begin{array}{c}\text { RTL } \\
(\mu \mathrm{l})\end{array}$ & $\begin{array}{c}\text { Linfocitos } \\
(\mu \mathrm{l})\end{array}$ & $\begin{array}{c}\text { Neutrofilos } \\
(\mu \mathrm{l})\end{array}$ & $\begin{array}{c}\text { Monocitos } \\
(\mu \mathrm{l})\end{array}$ & $\begin{array}{c}\text { Eosinofilos } \\
(\mu \mathrm{l})\end{array}$ \\
\hline $\begin{array}{c}\text { Control } \\
0(\mathrm{n}=3)\end{array}$ & $38,7( \pm 1,2)^{\mathrm{ab}}$ & $51,3( \pm 5)^{\mathrm{a}}$ & $3,3( \pm 1,2)^{\mathrm{a}}$ & $14533( \pm 4356)^{\mathrm{ab}}$ & $8821( \pm 1599)^{\mathrm{a}}$ & $4878( \pm 1381)^{\mathrm{a}}$ & $527( \pm 218)^{\mathrm{a}}$ & $82( \pm 87)^{\mathrm{a}}$ \\
$\begin{array}{c}\text { Inoculados } \\
\end{array}$ & & & & & & & & \\
$0(\mathrm{n}=9)$ & $40,3( \pm 1,6)^{\mathrm{a}}$ & $50,7( \pm 2)^{\mathrm{a}}$ & $3,1( \pm 1,1)^{\mathrm{a}}$ & $13772( \pm 3631)^{\mathrm{b}}$ & $7314( \pm 1033)^{\mathrm{a}}$ & $5478( \pm 1129)^{\mathrm{a}}$ & $550( \pm 220)^{\mathrm{a}}$ & $45( \pm 69)^{\mathrm{a}}$ \\
$7(\mathrm{n}=9)$ & $35,4( \pm 1,7)^{\mathrm{b}}$ & $55,8( \pm 8,1)^{\mathrm{a}}$ & $3,6( \pm 2,4)^{\mathrm{a}}$ & $15683( \pm 3116)^{\mathrm{ab}}$ & $8834( \pm 1678)^{\mathrm{a}}$ & $5680( \pm 1678)^{\mathrm{a}}$ & $906( \pm 360)^{\mathrm{a}}$ & $34( \pm 94)^{\mathrm{a}}$ \\
$14(\mathrm{n}=6)$ & $31,2( \pm 6,2)^{\mathrm{c}}$ & $57,3( \pm 3,5)^{\mathrm{a}}$ & $4,8( \pm 1,6)^{\mathrm{a}}$ & $18816( \pm 2946)^{\mathrm{a}}$ & $9000( \pm 753)^{\mathrm{a}}$ & $7276( \pm 941)^{\mathrm{a}}$ & $1913( \pm 809)^{\mathrm{b}}$ & $62( \pm 94)^{\mathrm{a}}$ \\
$21(\mathrm{n}=3)$ & $33,7( \pm 0,6)^{\mathrm{bc}}$ & $51,3( \pm 3,1)^{\mathrm{a}}$ & $4,7( \pm 2,3)^{\mathrm{a}}$ & $20216( \pm 4573)^{\mathrm{a}}$ & $9568( \pm 1415)^{\mathrm{a}}$ & $7413( \pm 1496)^{\mathrm{a}}$ & $3032( \pm 202)^{\mathrm{c}}$ & $66( \pm 121)^{\mathrm{a}}$ \\
\hline
\end{tabular}

Letras diferentes en sentido vertical indican diferencias significativas $(\mathrm{P}<0,05)$.

$\mathrm{RTL}=$ Recuento total de leucocitos.

Cuadro 2. Promedios $( \pm \mathrm{DE})$ del número de células mieloides (M), eritroides (E) y relación M: E obtenidas de los cerdos controles, sacrificados a los $0 \mathrm{dpi}(\mathrm{n}=3)$, y de los cerdos inoculados con el aislado chileno del vPRRS, sacrificados a los 7 $(n=3), 14(n=3)$ y $21(n=3)$ dpi.

Mean ( $\pm \mathrm{SD}$ ) numbers of myeloid (M), erythroid (E) and $\mathrm{M}$ :E ratio obtained from the control pigs, and pigs inoculated whit Chilean PRRSv isolates sacrificed $7(n=3), 14(n=3)$ and $21(n=3)$ dpi.

\begin{tabular}{cccc}
\hline DPI & Mieloide & Eritroide & Relación M:E \\
\hline Control & & & \\
0 & $253,3( \pm 5)^{\mathrm{a}}$ & $246,7( \pm 5)^{\mathrm{a}}$ & $1( \pm 0,04)^{\mathrm{a}}$ \\
Inoculados & & & \\
7 & $260,3( \pm 1,5)^{\mathrm{a}}$ & $239,7( \pm 1,5)^{\mathrm{a}}$ & $1,1( \pm 0,01)^{\mathrm{a}}$ \\
14 & $265,3( \pm 5,7)^{\mathrm{a}}$ & $234,7( \pm 5,7)^{\mathrm{a}}$ & $1,1( \pm 0,05)^{\mathrm{a}}$ \\
21 & $255,3( \pm 11,2)^{\mathrm{a}}$ & $244,7( \pm 11,2)^{\mathrm{a}}$ & $1,1( \pm 0,1)^{\mathrm{a}}$ \\
\hline
\end{tabular}

Letras diferentes en sentido vertical indican diferencias significativas $(\mathrm{P}<0,05)$.

\section{LESIONES HISTOPATOLOGICAS}

Organos linfoides: Los cerdos controles presentaron leve actividad mitótica y escasas necrosis aisladas en los folículos, mientras que en todos los cerdos inoculados se observó aumento de la actividad mitótica, necrosis linfoides aisladas, depleción leve y activación de centros germinales. Las lesiones variaron en distribución e intensidad con el transcurso de la infección. Los nódulos linfáticos mediastínicos presentaron las lesiones más intensas de todos los órganos linfoides evaluados y fueron evidentes a los 7 y $14 \mathrm{dpi}$, con mayor severidad a los 21 dpi al igual que lo observado con los nódulos linfáticos retrofaríngeos (figura 1). En la tonsila, las lesiones de mayor intensidad se registraron a los 7 y 14 dpi en el área folicular y se observó gran cantidad de detritus celulares, infiltración mononuclear de moderada intensidad y grupos de células necróticas dentro de las criptas. En el timo, las lesiones se observaron tanto en la corteza como en la médula con mayor intensidad a los $21 \mathrm{dpi}$. En

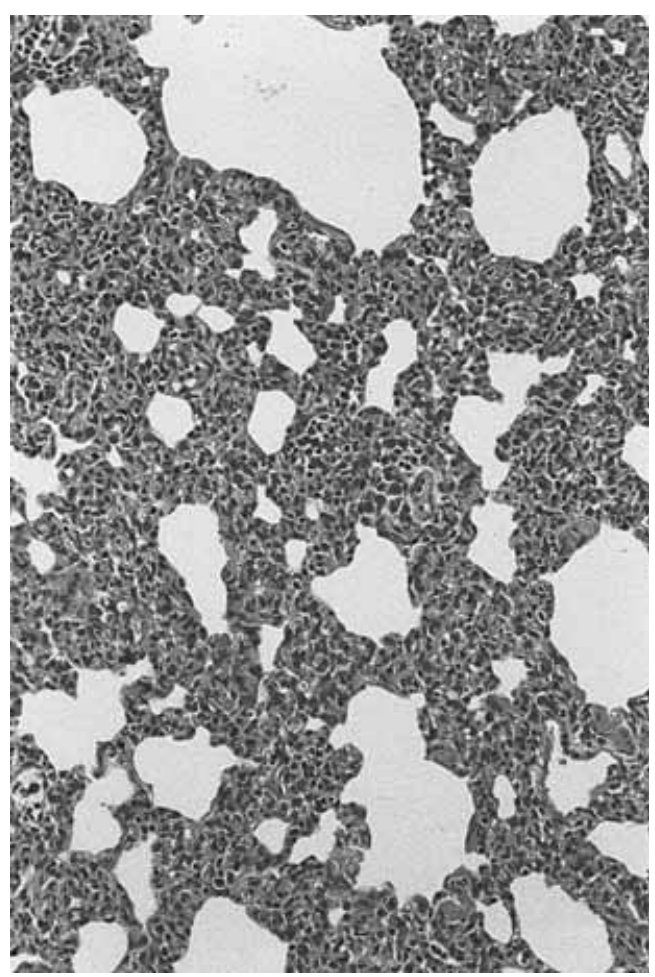

Figura 1. Nódulo linfático mediastínico: Múltiples células en necrosis con condensación densa de la cromatina $(\downarrow)$ y fragmentación nuclear $(\rightarrow)$ (7 dpi). H\&E, x1000.

Mediastinic lymph node: Multiple necrotic cells in the lymphoid follicle with dense condensation of the chromatin $(\triangleright)$ and nuclear fragmentation $(\rightarrow)$ (7 pid). H\&E, x1000.

el bazo se presentaron necrosis aisladas tanto en folículos como en vainas linfoides periarteriolares (PALS) y la mayor intensidad de las lesiones se observó a los 14 dpi (cuadro 3).

Organos respiratorios: Las lesiones en los cornetes nasales fueron similares en todos los cerdos, pero con distinta frecuencia e intensidad. Se observó rinitis carac- 
Cuadro 3. Lesiones histopatológicas encontradas en los órganos linfoides en los cerdos del grupo control e inoculados con el aislado chileno del vPRRS.

Histopathological lesions found in lymphoid organs of the control pigs and pigs inoculated a Chilean vPRRS isolate.

\begin{tabular}{|c|c|c|c|c|c|c|c|c|c|c|c|c|}
\hline & \multicolumn{12}{|c|}{ Días post inoculación } \\
\hline & \multicolumn{3}{|c|}{ CONTROL } & \multicolumn{3}{|c|}{7} & \multicolumn{3}{|c|}{14} & \multicolumn{3}{|c|}{21} \\
\hline & 1 & 2 & $\overline{3}$ & 1 & 2 & 3 & 1 & 2 & 3 & 1 & 2 & 3 \\
\hline \multicolumn{13}{|l|}{ Tonsila } \\
\hline Detritus en criptas & + & ++ & ++ & ++ & +++ & ++ & ++ & +++ & +++ & + & ++ & ++ \\
\hline Depleción en folículos & - & - & - & + & + & - & - & + & + & - & + & + \\
\hline Necrosis linfoide aisladas & + & + & + & ++ & +++ & + & ++ & ++ & ++ & + & + & ++ \\
\hline \multicolumn{13}{|l|}{ Timo } \\
\hline Infiltración de eosinófilos & + & + & + & ++ & ++ & + & ++ & +++ & +++ & ++ & ++ & ++ \\
\hline Necrosis linfoide & + & + & + & ++ & ++ & + & ++ & ++ & ++ & +++ & +++ & +++ \\
\hline \multicolumn{13}{|c|}{ Nódulo linfático retrofaringeo } \\
\hline \multicolumn{13}{|l|}{ Corteza } \\
\hline Depleción de folículos & - & - & - & - & - & - & - & - & - & - & - & - \\
\hline Necrosis linfoide & + & + & + & + & + & + & + & + & + & + & ++ & ++ \\
\hline \multicolumn{13}{|c|}{ Nódulo linfático mediastínico } \\
\hline \multicolumn{13}{|l|}{ Corteza } \\
\hline Depleción de folículos & - & - & - & + & + & - & - & + & + & + & + & ++ \\
\hline Necrosis linfoide & + & + & + & ++ & ++ & ++ & ++ & +++ & +++ & ++ & +++ & +++ \\
\hline \multicolumn{13}{|l|}{ Bazo } \\
\hline \multicolumn{13}{|l|}{ Folículos } \\
\hline Depleción & - & - & - & - & - & - & - & - & + & - & - & - \\
\hline Necrosis linfoide & + & + & + & ++ & ++ & ++ & +++ & +++ & +++ & ++ & ++ & ++ \\
\hline \multicolumn{13}{|l|}{$P A L S$} \\
\hline Depleción & - & - & - & - & - & - & - & - & + & - & - & - \\
\hline Necrosis linfoide & + & + & + & ++ & ++ & ++ & +++ & +++ & +++ & ++ & I++ & $1++$ \\
\hline
\end{tabular}

- Intensidad de la lesión: -: ausencia, +: leve, ++: moderado, +++: intenso.

- Números del 1 al 3 indican los diferentes cerdos durante cada necropasia.

- PALS: Vainas linfoides periarteriolares.

terizada por infiltración subepitelial multifocal de macrófagos, linfocitos y algunos polimorfosnucleares en la lámina propia con pérdida de la integridad epitelial e infiltración leucocitaria de éste, con mayor intensidad a los 14 dpi.

Todos los cerdos inoculados presentaron neumonía intersticial caracterizada por infiltración septal de células mononucleares y la mayor intensidad se observó a los 14 dpi (figura 2 y 3). Además, se encontró aumento de MAPs, necrosis celulares aisladas y sincicios en los septos y alvéolos, áreas focales de colapso e infiltración linfoide perivascular leve. A los 21 dpi, la infiltración de los septos fue menos intensa y se observó hiperplasia del tejido linfoide peribronquiolar y escaso exudado en los conductos (cuadro 4).

Hígado: Se observó hiperplasia e hipertrofia de las células de Küffer e infiltrados mononucleares focales intralobulillares y periportales, siendo más intensas a los $14 \mathrm{dpi}$.
Corazón: Las alteraciones en este órgano se limitaron a cuadros de miocarditis focal leve con infiltrado mononuclear de baja intensidad en el intersticio alrededor de vasos y entre las fibras musculares en un cerdo a los 7 dpi, en los tres cerdos a los 14 dpi y en dos cerdos a los 21 dpi.

Sistema nervioso central: En el encéfalo se observaron manguitos perivasculares no purulentos de tipo linfocitario tanto en la sustancia blanca como en la gris de cerebro y cerebelo (figura. 4). Estas lesiones no fueron constantes, observándose en dos cerdos en cerebelo a los $7 \mathrm{dpi}$, en tres cerdos en cerebro y en dos cerdos en cerebelo a los 14 dpi y en un cerdo en cerebro y cerebelo a los 21 dpi. En las meninges también se observaron discretos manguitos en un cerdo a los $7 \mathrm{dpi}$, en tres cerdos a los 14 dpi y en un cerdo a los 21 dpi. 
Cuadro 4. Lesiones histológicas encontradas en el pulmón de los cerdos controles e inoculados con el aislado chileno del vPRRS. Histopathological lesions found in lungs of the control pigs and pigs inoculated with a Chilean vPRRS isolate.

\begin{tabular}{|c|c|c|c|c|c|c|c|c|c|c|c|c|c|c|c|}
\hline \multirow[t]{2}{*}{$\begin{array}{l}\text { Días post } \\
\text { inoculación }\end{array}$} & \multicolumn{3}{|c|}{ Neumonitis } & \multicolumn{3}{|c|}{$\begin{array}{l}\text { Necrosis aisladas en } \\
\text { células septales }\end{array}$} & \multicolumn{3}{|c|}{$\begin{array}{l}\text { Aumento } \\
\text { MAPs }\end{array}$} & \multicolumn{3}{|c|}{$\begin{array}{l}\text { Infiltrado } \\
\text { Perivascular }\end{array}$} & \multicolumn{3}{|c|}{$\begin{array}{c}\text { Hiperplasia Tejido } \\
\text { Linfoide } \\
\text { Peribronquiolar }\end{array}$} \\
\hline & 1 & 2 & 3 & 1 & 2 & 3 & 1 & 2 & 3 & 1 & 2 & 3 & 1 & 2 & 3 \\
\hline \multicolumn{16}{|c|}{ CONTROL } \\
\hline $\begin{array}{r}0 \\
\text { INOCULA }\end{array}$ & \multicolumn{14}{|c|}{ INOCULADOS } & + \\
\hline 7 & + & ++ & +++ & + & + & - & + & - & - & - & - & - & - & + & ++ \\
\hline 14 & ++ & +++ & +++ & + & + & - & +++ & + & + & + & + & - & + & ++ & ++ \\
\hline 21 & ++ & ++ & ++ & - & + & - & + & + & + & + & + & + & ++ & ++ & +++ \\
\hline
\end{tabular}

- Intensidad de la lesión: -: ausencia; +: leve; ++: moderado; +++: intenso.

- Números del 1 al 3 indican los diferentes cerdos durante cada necropsia.

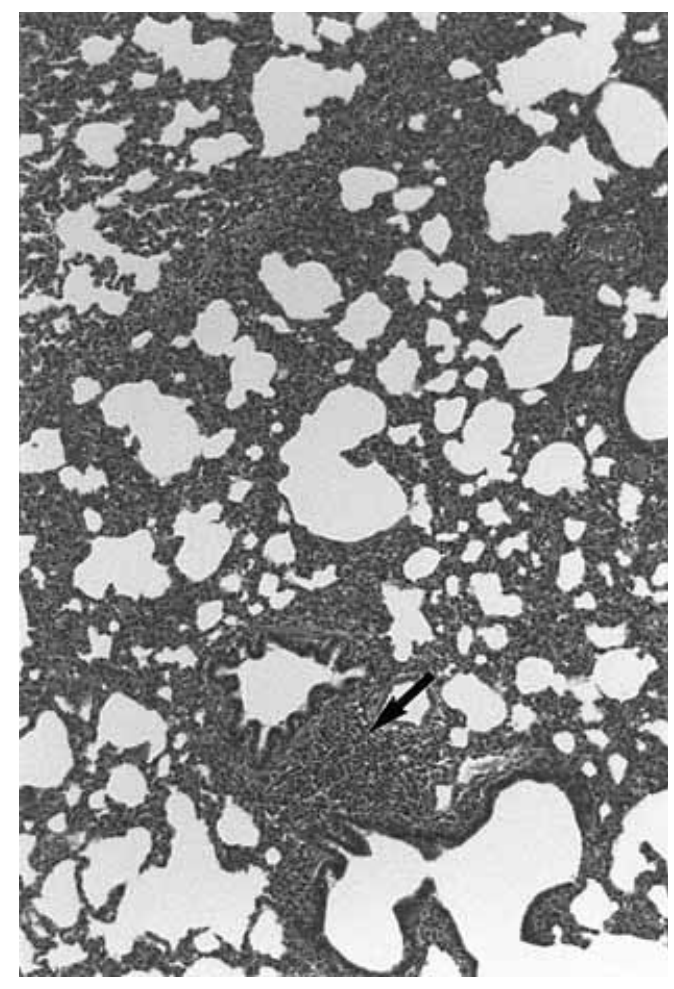

Figura 2. Pulmón: Neumonía intersticial con infiltrado mononuclear en septos e hiperplasia del tejido linfoide peribronquial $\rightarrow$ ( $(21$ dpi). H\&E, x100.

Lung: Interstitial pneumonia with mononuclear septal infiltrated and peribronchial hyperplasia of lymphoid tissue $(\rightarrow)(21$ pid). H\&E, x100.

\section{DISCUSION}

El aislado chileno del vPRRS produjo alteraciones hematológicas evidentes entre los 7 y $21 \mathrm{dpi}$, caracterizadas principalmente por disminución del VG y aumento de leucocitos asociado a un incremento de monocitos. Las infecciones bacterianas o virales pue-

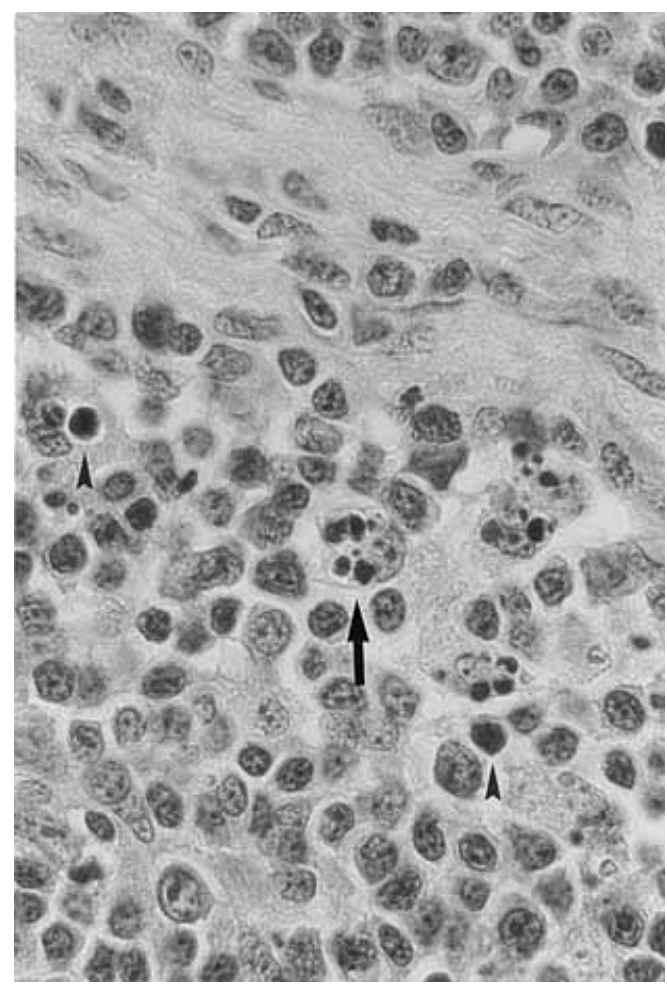

Figura 3. Pulmón: Engrosamiento de septos por infiltrado de macrófagos y linfocitos (7 dpi). H\&E x400.

Lung: Septal thickening by infiltration of macrophages and lymphocytes (7 pid). H\&E x400.

den causar anemia, producto de las reacciones inflamatorias que ejercen estos agentes en los tejidos (Stockham 2000), la cual estaría mediada por citoquinas secretadas durante la inflamación, lo que trae como consecuencia una menor disponibilidad de hierro, disminución en la supervivencia de los eritrocitos y menor respuesta de la médula ósea a la eritropoyetina (Trevor 


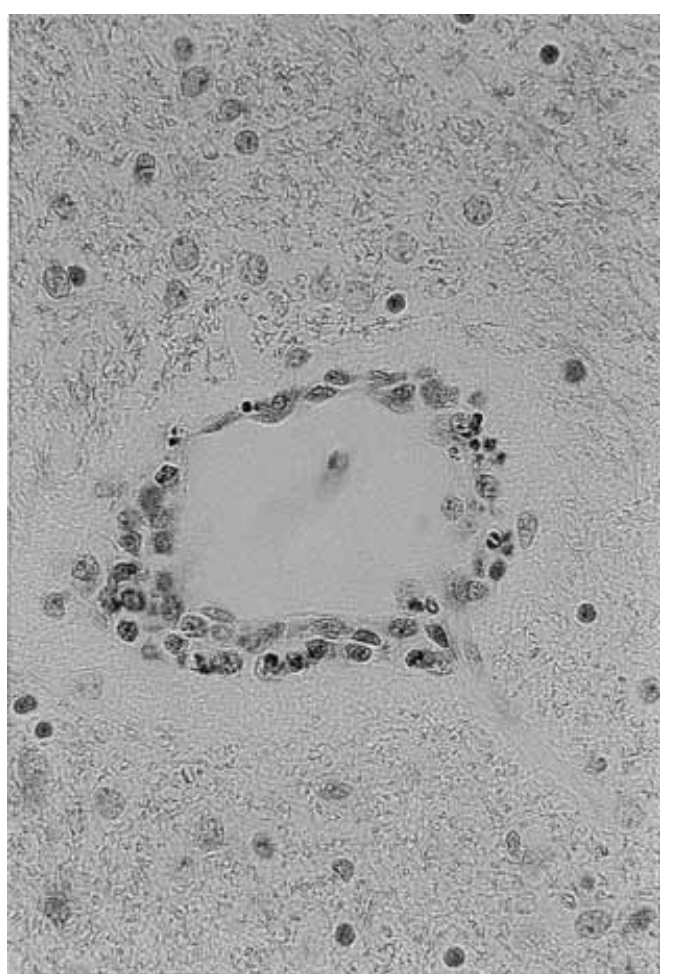

Figura 4. Cerebelo: Manguito perivascular no purulento (14 dpi). H\&E x400.

$H \& \mathrm{E} \times 400$.

Cerebellum: Non- suppurative perivascular cuffs (14 pid)

y Harrus 2000). Al respecto, se ha demostrado que los cerdos infectados con el vPRRS presentan un aumento de citoquinas inflamatorias, liberadas desde los macrófagos (Feng y col 2003, Labarque y col 2003), las cuales podrían iniciar y mediar la disminución del VG. A su vez, estos cambios también podrían explicarse a través del efecto directo sobre la médula ósea que ejercería el vPRRS como consecuencia de la replicación viral, lo que produciría la inhibición de los precursores eritroides; sin embargo, la presencia del vPRRS en la médula ósea no ha sido demostrada eficientemente (Halbur y col 2002), por lo cual este mecanismo no está claramente establecido.

El aumento del fibrinógeno en la sangre es un claro indicador de inflamación aguda, ya que en estos procesos la medición de proteínas de fase aguda se presentan aumentadas (Thorn 2000). En este estudio no se presentó un aumento significativo en los niveles de fibrinógeno en los cerdos inoculados con el vPRRS, aunque sí fue posible observar una tendencia a aumentar entre los $14 \mathrm{y}$ 21 dpi. Por otra parte, tampoco se observaron alteraciones a nivel de médula ósea, lo cual difiere con lo descrito por Halbur y col (2002), quienes observaron un aumento considerable en la relación M:E desde los 3 dpi en cerdos infectados con aislados norteamericanos de alta virulencia.
El aumento en el recuento de leucocitos totales asociado a un incremento en los monocitos circulantes podría ser explicado por la mayor demanda de estas células asociado a la replicación viral en macrófagos y el efecto citolítico que este ejerce sobre ellas. Rossow y col (1995) establecen que la afinidad del vPRRS por macrófagos alveolares gatilla el reclutamiento de otras poblaciones de macrófagos. Para que ello ocurra, necesariamente debería aumentar la población de monocitos circulantes; sin embargo, se ha demostrado que el virus causa leucopenia entre los 3 y 7 dpi, debido a una disminución en los linfocitos, monocitos y neutrófilos (Rossow y col 1994, Nielsen y Bøtner 1997, Halbur y col 2002). Esto resulta difícil de precisar en esta experiencia, ya que no se realizaron hemogramas entre los 0 y 7 dpi.

Por otra parte, las lesiones externas observadas en la necropsia resultan similares a las descritas por Rossow y col (1995) y Halbur y col (1996b) en cerdos inoculados con aislados norteamericanos del vPRRS, aunque el aislado chileno no presenta la regularidad e intensidad de las lesiones observadas con aislados de alta virulencia descritas por estos autores.

Histopatológicamente, los órganos linfoides presentaron activación de centros germinales e incremento de actividad mitótica a los 14 y 21 dpi. Por otra parte, se observaron en forma constante necrosis aisladas en folículos y tejido linfoide difuso, las cuales han sido descritas ampliamente como fenómenos de apoptosis; sin embargo, se ha demostrado que las células que presentan apoptosis no necesariamente son aquellas células infectadas por el virus, ya que las citoquinas liberadas durante los procesos inflamatorios en los tejidos infectados también pueden inducir apoptosis (Sur y col 1998, Labarque y col 2003). Con respecto a los órganos respiratorios, en las vías aéreas se observó rinitis, especialmente en los cerdos sacrificados a los 14 dpi, lo cual concuerda con lo descrito por Pol y col (1991), Rossow y col (1995) Halbur y col (1995) y Halbur y col (1996b). Los macrófagos asociados a la mucosa nasal sería uno de los sitios primarios de replicación del vPRRS (Rossow 1998), lo cual explicaría las lesiones observadas en los cornetes nasales de los cerdos de esta experiencia. En cuanto a las lesiones pulmonares, en este estudio no se observaron lesiones macroscópicas evidentes, aunque algunos autores señalan alteraciones caracterizadas principalmente por cambios de color y firmeza del parénquima pulmonar en distintos lóbulos (Rossow y col 1995, Halbur y col 1996b). La ausencia de lesiones macroscópicas específicas en los pulmones de los cerdos infectados concuerda con lo descrito por Nodelijk (2002), quien indica que las lesiones son fundamentalmente histológicas en este órgano, a no ser que se presenten infecciones bacterianas secundarias (Rossow y col 1995), aunque la intensidad de las neumonías puede variar, según la virulencia de los distintos aislados virales (Halbur y col 1996b). Por otra parte, las lesiones mi- 
croscópicas encontradas en los pulmones en esta experiencia se caracterizaron por presentar un típico patrón de neumonía intersticial como la descrita ampliamente por diversos autores (Pol y col 1991, Collins y col 1992, Rossow y col 1994, Rossow y col 1995, Halbur y col 1995, Halbur y col 1996b). Las lesiones pulmonares se presentaron en todos los cerdos inoculados siendo más intensas a los $14 \mathrm{dpi}$, lo que concuerda con lo indicado por otros autores, quienes describen la presentación de neumonía intersticial entre los 3 y $28 \mathrm{dpi}$, con mayor intensidad alrededor de los 10 dpi (Collins y col 1992, Rossow y col 1995, Halbur y col 1996b).

Los infiltrados mononucleares encontrados en hígado y corazón se deberían a la migración de macrófagos infectados a estos tejidos (Rossow 1998), sin embargo, la leve intensidad de las lesiones que se observó en estos órganos sugiere que la replicación viral en ellos resultaría escasa o nula.

En este estudio fue posible observar encefalitis y meningitis de leve intensidad, las cuales no se presentaron de forma constante en todos los cerdos, lo cual difiere de lo descrito en estudios previos realizados con cepas altamente virulentas donde las lesiones encefálicas afectaban a todos los cerdos infectados con intenso infiltrado perivascular (Collins y col 1992, Rossow y col 1995, Rossow y col 1996b, Rossow y col 1999, Halbur y col 1995, Halbur y col 1996b). A su vez, está demostrado que el vPRRS puede replicar en células de la microglia del cerebro (Molitor y col 1997) y causar lesiones y signos clínicos compatibles con meningoencefalitis (Rossow y col 1999); sin embargo, la intensidad de las lesiones del encéfalo presentan claras variaciones, según la virulencia de los distintos aislados como lo demostraron Halbur y col (1996b). Esto podría explicar la presentación de lesiones encefálicas leves e inconstantes en los cerdos inoculados con el aislado chileno.

De acuerdo a los estudios de patogenicidad y virulencia realizados por varios autores (Collins y col 1992, Rossow y col 1995, Rossow y col 1996b, Rossow y col 1999, Halbur y col 1995, Halbur y col 1996b), las lesiones observadas en esta experiencia demuestran que el cuadro corresponde a una infección multisistémica, aunque la intensidad leve e inconstante de las lesiones macroscópicas y microscópicas de órganos linfoides, corazón y encéfalo sugieren que el aislado chileno del vPRRS sería de baja virulencia.

\section{RESUMEN}

El presente trabajo se planteó con el fin de caracterizar las manifestaciones hematológicas y lesiones de cerdos inoculados con el aislado chileno del vPRRS. Se utilizaron 12 cerdos de 3 semanas de edad divididos en 4 grupos de 3 animales cada uno, uno de los cuales correspondió al grupo control que fueron sacrificados a los 0 días postinoculación (dpi), y los 3 grupos restantes a los cerdos inoculados que fueron sacrificados a los
7, 14 y 21 dpi. Durante cada muestreo se recolectó sangre para hemogramas y al sacrificio se determinaron las lesiones macroscópicas y se tomaron muestras para frotis de médula ósea y para histopatología. Los resultados de este estudio revelaron alteraciones hematológicas caracterizadas por descenso del volumen globular $(\mathrm{P}<0,05)$ y aumento en el recuento de leucocitos totales $(\mathrm{P}<0,05)$ asociado a un aumento de los monocitos. La médula ósea no presentó variaciones en la relación de células mieloides y eritroides $(\mathrm{P}>0,05)$. Las lesiones macroscópicas fueron de leve intensidad y caracterizadas principalmente por la presencia de conjuntivitis, edema periocular y leve aumento de tamaño de los linfonódulos. Las lesiones microscópicas se caracterizaron por la presencia de neumonía intersticial, depleción y necrosis linfoide en órganos linfoides, rinitis, hepatitis, miocarditis y encefalitis no purulenta. Estos hallazgos sugieren que el aislado chileno del PRRSv correspondería a uno de baja virulencia.

\section{AGRADECIMIENTOS}

Los autores desean agradecer a las autoridades del Servicio Agrícola y Ganadero por el apoyo al proyecto y al Dr. Fernando Osorio de la Universidad de Nebraska, Lincoln, USA, por el asesoramiento virológico.

\section{REFERENCIAS}

Benfield D A, J E Collins, S A Dee, P G Halbur, H S Hoo, K M Lager, W L Mengeling, M P Murtaugh, K D Rossow, G W Stevenson, J J Zimmerman. 1999. Porcine reproductive and respiratory syndrome virus. En: B. E. Straw, S. D'Allaire, W. L. Mengeling, D. J. Taylor (eds). Diseases of Swine. Pp 201-232. Ames, Iowa.

Cavanagh, D. 1997. Nidovirales: a new order comprising Coronaviridae and Arteriviridae. Arch Virol 142, 629-633.

Christianson, W T, J E Collins, D A Benfield, L Harris, D E Gorcyca, D W Chladek, R B Morrison, H S Joo. 1992. Experimental reproduction of swine infertility and respiratory syndrome in pregnant sows. Am J Vet Res 53, 485-488.

Collins J E, D A Benfield, W T Christianson, L Harris, J C Hennings, D P Shaw, S M Goyal, S McCullough, R B Morrison, H S Joo. 1992. Isolation of swine infertility and respiratory syndrome virus (isolate ATCC VR-2332) in North America and experimental reproduction of the disease in gnotobiotic pigs. J Vet Diagn Invest 4, 117-126.

Done S H, D J Paton. 1995. Porcine reproductive and respiratory syndrome: Clinical disease, pathology and immunosuppression. Vet Rec 136, 32-35.

Feng W H, S M Laster, M Tomkins, T Brown, J S Xu, C Altier, W Gomez, D Banfield, M B McCaw. 2001. In utero infection by porcine reproductive and respiratory syndrome virus is sufficient to increase susceptibility of piglets to challenge by Streptococcus suis type II. J Virol 75, 48894895.

Feng W H, M B Tomkins, J S Xu, H X Zhang, M B McCaw. 2003. Analysis of constitutive cytokine expression by pigs infected in utero with porcine reproductive and respiratory syndrome virus. Vet Immunol Immunopathol 94, 35-45.

Goyal, S M. 1993. Porcine reproductive and respiratory syndrome. J Vet Diag Invest 5, 656-664. 
Halbur P G, L D Miller, P S Paul, X J Meng, E L Huffman J J Andrews. 1995. Immunohistochemical identification of porcine reproductive and respiratory syndrome virus antigen in the heart and lymphoid system of three-weekold calostrum-deprived pigs. Vet Pathol 32, 200-204.

Halbur P G, P S Paul, M L Frey, J Landgraf, K Eernisse, X J Meng, J J Andrews, M A Lum, J A Rathje, 1996. Comparison of the antigen distribution of two US porcine reproductive and respiratory syndrome virus isolates with that of the Lelystad virus. Vet Pathol 33, 159-170.

Halbur P G, P S Paul, X J Meng, M A Lum, J J Andrews, J A Rathje. $1996^{\mathrm{b}}$. Comparative pathogenicity of nine US porcine reproductive and respiratory syndrome (vPRRS) isolates in a five-week-old cesaream-derived, calostrumdeprived pig model. $J$ Vet Diag Invest. 8, 11-20.

Halbur P G, P S Paul, X J Meng, M A Lum, J J Andrews, J A Rathje. $1996^{\mathrm{b}}$. Comparative pathogenicity of nine US porcine reproductive and respiratory syndrome (vPRRS) isolates in a five-week-old cesaream-derived, calostrumdeprived pig model. J Vet Diag Invest. 8, 11-20.

Labarque G S, Van Gucht, H Nauwynck, K Van Reeth, M Pensaert. 2003. Apoptosis in the lungs of pigs infected with porcine reproductive and respiratory syndrome virus and associations with the production of apoptogenic cytokines. Vet Res 34, 249-260.

Molitor T W, E M Bautista, C S Choi. 1997. Immunity to vPRRS: Double-edged sword. Vet Microbiol 55, 265-276.

Nielsen, J, A Bøtner. 1997. Hematological and immunological parameters of $41 / 2$ month old pigs infected with PRRS virus. Vet Microbiol 55, 289-294.

Nodelijk G. 2002. Porcine reproductive and respiratory syndrome (PRRS) with special reference to clinical aspects and diagnosis: a review. Vet $Q 24,95-100$.

Pol J M, A J Van Dijk, G Wensvoort, C Terpstra. 1991. Pathological, ultrastructural, and immunohistochemical changes caused by Lelystad virus in experimentally induced infections of mystery swine disease (synonym: porcine epidemic abortion and respiratory syndrome (PEARS). Vet $Q 13,137-143$.

Rossow K D, E M Bautista, S M Goyal, T W Molitor, M P Murtaugh, R B Morrison, D A Benfield, J E Collins. 1994. Experimental porcine reproductive and respiratory syndrome virus infection in one-, four-, and 10-week-old pigs. J Vet Diag Invest 6, 3-12.

Rossow K D, J E Collins, S M Goyal, E A Nelson, J Christopher-Hennings D A Benfield. 1995. Pathogenesis of porcine reproductive and respiratory syndrome virus infection in gnotobiotic pigs. Vet Pathol 32, 361-373.

Rossow K D, D A Benfield, S M Goyal, E A Nelson, J Christopher-Hennings J E Collins. 1996ª Chronological immunohistochemical detection and localization of porcine reproductive and respiratory syndrome virus in gnotobiotic pigs. Vet Pathol 33, 551-556.

Rossow K D, K L Laube, S M Goyal, J E Collins. 1996'b. Fetal microscopic lesions in porcine reproductive and respiratory syndrome virus-induced abortion. Vet Pathol 33, 95-99.

Rossow K D. 1998. Porcine reproductive and respiratory syndrome. Vet Pathol 35, 1-20.

Rossow K D, J L Shivers, P E Yeske, D D Polson, R R Rowland, S R Lawson, M P Murtaugh, E A Nelson, J E Collins. 1999. Porcine reproductive and respiratory syndrome virus infection in neonatal pigs characterized by marked neurovirulence. Vet Rec 144, 444- 448.

Ruiz A, L Cuevas, J Naranjo. 2003. Chile: Program to eradicate PRRS virus. En: J. Zimmerman, K. J. Yoon (eds). 2003 PRRS Compendium. Pp 221-222. Ames, Iowa.

Schalm, O W, N C Jain, E J Carroll. 1975. Material and Methods for the study of the blood, icluding brief comments on factors to be considered in interpretation. En: Schalm O W, N C Jain, E J Carroll. Veterinary Hematology, p 15 81. Philadelphia.

Shin, J, J Torrison, C S Choi, S M Gonzalez, B G Crabo, T W Molitor. 1997. Monitoring of porcine reproductive and respiratory syndrome virus infection in boars. Vet Microbiol 55, 337-346.

Stockham S L. 2000. Anemia associated whit bacterial and viral infections. En: B E Feldman, J G Zinkl, N C Jain (eds). Schalm's Veterinary Hematology. Pp 163-168. Philadelphia.

Sur J H, A R Doster, J S Christian, J A Galeota, R W Wills, J J Zimmerman, F A Osorio. 1997. Porcine reproductive and respiratory syndrome virus replicates in testicular germ cells, alters spermatogenesis, and induces germ cell death by apoptosis. $J$ Virol 71, 9170-9179.

Sur J H, A R Doster, F A Osorio. 1998. Apoptosis induced in vivo during acute infection by porcine reproductive and respiratory syndrome virus. Vet Pathol 35, 506-514.

Thorn C E. 2000. Normal hematology of pig. En: B E Feldman, J G Zinkl, N C Jain (eds). Schalm's Veterinary Hematology, pp 1089-1095. Philadelphia.

Trevor W, S Harrus. 2000. Anemia of inflammatory disease. En: B E Feldman, J G Zinkl, N C Jain (eds). Schalm's Veterinary Hematology. Pp 205-209. Philadelphia.

Van Reeth K., G Labarque, H Nauwynck, M Pensaert. 1999. Differential production of proinflammatory cytokines in the pig lung during different respiratory virus infection: correlations with pathogenicity. Res Vet Sci 67, 47-52.

Wensvoort G, C Terpstra, J M Pol, E A Ter Laak, M Bloemraad, E P de Kluyver, C. Kragten, L Van Buiten, A Den Besten, F Wagenaar. 1991. Mystery swine disease in The Netherlands: the isolation of Lelystad virus. Vet $Q 13$, 121-130. 